\title{
Effect of Tactile Stimulation Pulse Characteristics on Sensation Threshold and Power Consumption
}

\author{
Enrico Nunziata, Claudio Perez, Ed Jarmul, \\ Leo E. Lipetz, and Herman R. Weed \\ Biomedical Engineering Center \\ The Ohio State University \\ 2015 Neil Ave. \\ Columbus, OH 43210 \\ (Received 2/1/88; Revised $11 / 10 / 88$ )
}

\begin{abstract}
The psychophysical responses of human subjects to vibratory tactile stimulation of the skin were investigated experimentally. The parameters of the waveform important to the minimization of power consumed by the tactile array of electromechanical vibrators and the maximization of the skin sensitivity to the stimulus were explored to develop optimum stimulation. Parameters investigated included the amplitude, frequency, and duty cycle of the current waveform used to drive the vibrators as well as the number of pulses per stimulating burst and the recovery time between bursts. Graphical techniques were used to determine the optimal combination of the parameters which gave a stimulus that excited the skin to above tactile threshold while maintaining at a relative minimum the power required for the stimulus. The optimal stimulation waveform contains a burst of 10 rectangular pulses of $4 \%$ duty cycle separated by a period of nonstimulation of $2 \mathrm{~s}$. Such a waveform can elicit a sensitivity of $29.4 \mathrm{~mA}^{-1}$ consuming only $55 \mu \mathrm{W}$ of power.
\end{abstract}

Keywords-Tactile stimulation, Tactile sensation, Sensitivity, Power consumption.

\section{INTRODUCTION}

A Tactile Vision Information System (TVIS) is a device that allows a blind or visually impaired individual to gain information about his or her environment. The basic function of the TVIS is the acquisition of an optical image with a videocamera, the processing of this image to match its resolution to that of a vibratory tactile array (VTA), and the transformation of the image or some portion of the image via the VTA, into a vibratory pattern on a specific region of the skin.

In the development of the TVIS, it is desired to minimize the power required to operate the vibratory array, both to reduce the heat produced at the array and to allow the array to be run from a portable battery pack. Such a minimization requires that the characteristics of the tactile stimulus be matched to the excitation response

Acknowledgment - This work was supported by a grant from R.O.S.E. Foundation, Canton, Ohio, and the American Council for the Blind.

Address correspondence to Enrico Nunziata, Piazza Cattaneo, 21/B 10137, Turino, Italy. 
characteristics of the tactile receptors of the skin. The stimulation can be provided with different types of devices which either produce electrical stimulation (1) (injecting current into the skin) or convert a current pattern into a vibratile one in the case of the tactile vibrators (6). The power associated with the stimulus depends on the amount of current to be delivered to the vibrators in order to elicit the response of the tactile system. The current required for a vibrator is several times higher than that used in direct skin electrical stimulation. However, the electromechanical stimulation is safer and generally more acceptable to the user.

In this study, the authors investigated various stimulation sequences, for an electromechanical vibrator, which produce sufficient energy to elicit tactile sensation. The ultimate objective was to find the waveform, or combination of waveforms, which produce the maximum tactile sensitivity with minimum power requirements. To accomplish this, a series of experiments were carried out to investigate the interrelation between parameters and to provide background information as a basis for logical conclusions. The results of these experiments led to the definition of a stimulating waveshape that activates the tactile sense while minimizing power consumption.

\section{MATERIALS AND METHODS}

\section{The Derivation of the Stimulation Waveform}

In preceding studies $(1,3,6)$ different waveforms (sinusoidal, triangular, and rectangular) were used to excite the tactile system. Several studies $(2,3,7)$ report the Pacinian corpuscles (PCs) to be the most responsive transducers in the tactile system in the vicinity of $250 \mathrm{~Hz}$ stimulation frequency. For lower stimulation frequencies $(<100 \mathrm{~Hz})$, the non-Pacinian receptors' response needs to be considered in defining the total response of the tactile system (13). Different researchers $(2,5)$ have investigated the mechanical as well as the mechano-to-neural transduction process in the PCs. The most important result produced by these studies is the nonlinear relationship between the magnitude of the response of the PCs and the slope of the onset and offset of the stimulating waveform. The greater the slope of the exciting waveform, the higher the response of the PCs (2). Once the slope of the exciting waveform is such that it can elicit the tactile sensor response, then the threshold becomes a function of the skin displacement, which is related to the exciting waveform amplitude $(4,10)$.

Sato (11), in his study on the response of the PCs to sinusoidal vibrations (tactile stimuli), reported that at $37^{\circ} \mathrm{C}$, the low threshold optimal frequency is approximately $300 \mathrm{~Hz}$. Some years later, Verrillo (12), exciting the skin with mechanical vibrations (sinusoidal waveforms), found that the minimum threshold for tactile sensation is reached at a vibration frequency of $250 \mathrm{~Hz}$.

The other property of the tactile system included in this study was adaptation. Adaptation can be defined as the reduced response of a sensor to a stimulus following exposure to a continued stimulus. To recover the skin receptors' maximum response, it was proposed to introduce a period of nonstimulation between two successive bursts of stimulation. If this period is well chosen (i.e., if it is long enough for the tactile sensors to recover their initial sensitivity), their response to the onset of the subsequent stimulation will be increased.

Based on the concepts in the above paragraph, it was proposed to use a vibrational stimulus whose shape is rectangular and whose fundamental frequency is $250 \mathrm{~Hz}$ in 


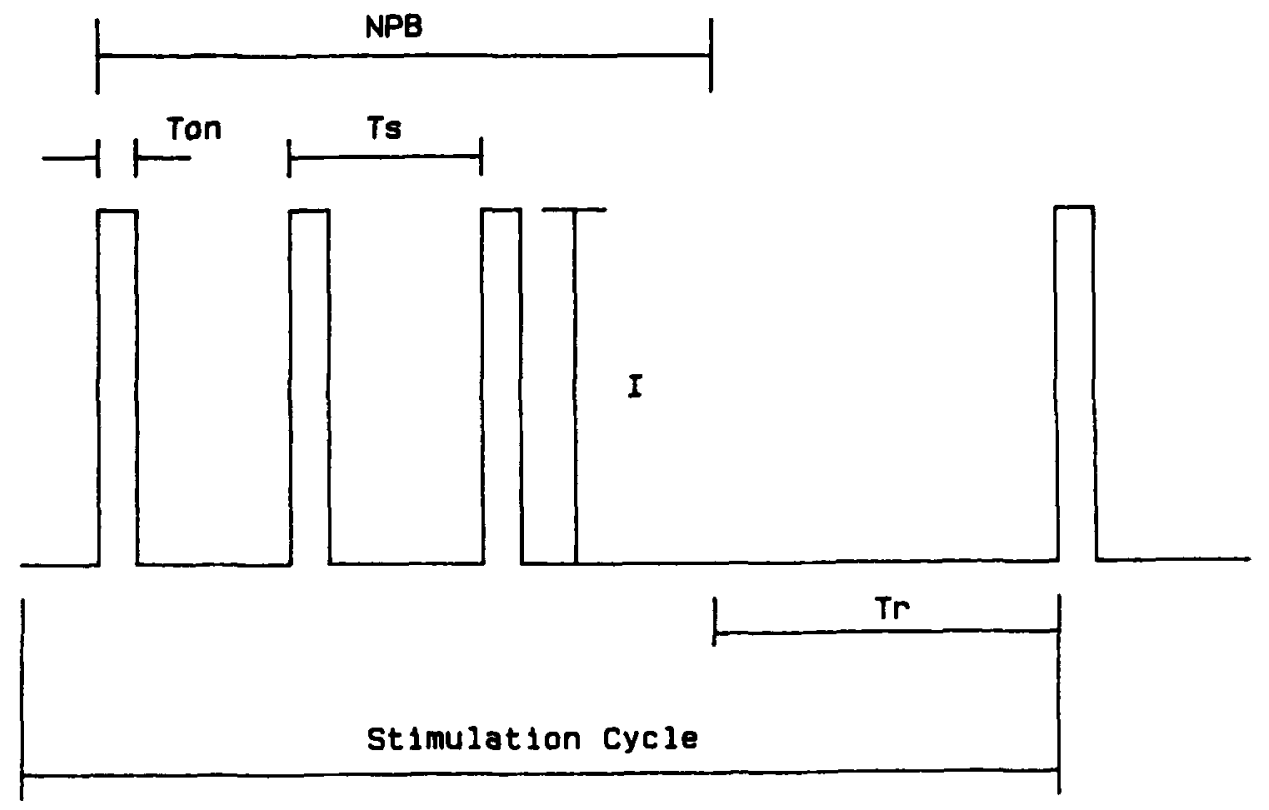

FIGURE 1. Stimulation sequence: current (ordinate) vs. time (abscissa). See text for the symbol definitions.

order to meet both the slope and frequency requirements (see Fig. 1). The width and amplitude of the pulse were made variable in order to investigate the effect of pulsewidth reduction on the tactile threshold and on the power consumption. Moreover, a period of nonstimulation between two consecutive bursts of pulses was introduced to allow the sensory transducer to recover from adaptation (see Fig. 1). Since the minimum recovery time period, and the number of pulses/burst which gave the greatest ratio of stimulation to sensitivity reduction were not known, these two quantities were also varied. The parameters characterizing the exciting sequence used are the following (refer to Fig. 1):

1. rectangular pulse amplitude $(I)$,

2. pulse frequency $\left(f_{p}=1 / T_{s}\right)$,

3. number of pulses per burst during the stimulating period (NPB),

4. pulse duty cycle $(D C$, fraction of the frequency period during which the current pulse flows; $D C=T_{\text {on }} / T_{s}$ ),

5. recovery time ( $T_{r}$, period between two bursts in which no current flows).

\section{Sensitivity and Power: Theoretical Considerations}

It was decided to investigate two types of sensation: (a) the level at which the subjects could first feel that something was touching their skin (defined as first sensation), and (b) the level at which they could recognize a straight line pattern touching the skin (defined as line sensation).

Sensitivity to the stimulation is defined as the inverse of the stimulus intensity; here that is, the inverse of the peak current to the vibrator to just reach the thresh- 
old for one of the above tactile sensations. If $I$ is the value of the peak current at the threshold for tactile sensation, the sensitivity can be expressed with Eq. 1. The specific nonlinearities of the stimulation current to vibrator motion are included in these definitions:

$$
S=1 / I \quad[1 / \mathrm{A}]
$$

The average power ${ }^{1}$ associated with the stimulating sequence of Fig. 1 at any given sensitivity is equal to:

$$
\begin{aligned}
E(P) & =(I \cdot V) \cdot\left(f_{p}^{-1} \cdot N P B \cdot D C\right) /\left(f_{p}^{-1} \cdot N P B+T_{r}\right) \\
& =(I \cdot V) \cdot(N P B \cdot D C) /\left(f_{p} \cdot T_{r}+N P B\right) \quad[\mathrm{W}]
\end{aligned}
$$

where $I=$ current through the vibrator at threshold for tactile sensation and $V=$ voltage drop across the vibrator at threshold for tactile sensation.

From Eq. 2 it is possible to determine the influence of each of the stimulating pattern's parameters on the power required for a threshold stimulus. The previous affirmation assumes the vibrator to be a pure resistor. The latter is true because at the frequency of interest $(250 \mathrm{~Hz})$ the vibrator behaves primarily as a resistive component (8) (see Fig. 2b, where $R=50 \mathrm{Ohm}$ and $L=7 \mathrm{mH}$ ). At higher frequencies the inductive component can still be negligible while maintaining an error in power computation less than $10 \%$.

The peak current also depends on the parameters defining the stimulating pattern shown in Fig. 1, but the amplitude of the peak current for threshold sensation can only be determined psychophysically. This is because psychological factors, such as subject's ability to perceive tactile stimulation, and physiological factors, such as age and the structure of the skin, affect tactile threshold. To evaluate the peak current at the threshold for tactile sensation, experimental tests were run, and statistical tools were used to determine which of the waveform parameters had the most influence on the peak current at the threshold for tactile sensation. Once the peak current was determined, the power was computed using Eq. 2 .

\section{The Experimental Design}

The problem relates to three independent variables $\left(T_{r}, D C, N P B\right)$ and one dependent variable $(I)$. Each independent variable has its own range which was chosen at the beginning of the test either intuitively or according to physiological requirements. Because of the three independent variables, the experimental design would involve an unnecessarily large number of tests if all the variables were tested at once. In order to avoid biasing the results along one path in the four-dimensional plane, for each experiment, two variables were kept constant and the third allowed to vary over some predetermined range. In this way more paths within the same plane were investigated.

In order to proceed logically from one test to the next, reducing the number of

\footnotetext{
${ }^{1}$ The average power is defined as the power consumed during a stimulation cycle (i.e., burst plus recovery time interval).
} 
variables and selecting the optimum set of the parameters, a test criterion was developed. The criterion chosen was:

1. Continue to increase/decrease a variable as long as:

a. Power decreases by $10 \%$ or more and

b. Sensitivity remains within $10 \%$ of the maximum value observed

2. Whenever a or $b$ is violated, choose last value as "optimum" in terms of minimum power and highest sensitivity for that test.

The specifications, constraints, and assumptions considered for each of the stimulation sequence parameters were:

Recovery time. Pacinian corpuscles, when directly stimulated, can respond with $95 \%$ of their original response, if a second stimulation is delivered after a delay of $40 \mathrm{~ms}$ (3). Since no study has been done on the effect of $T_{r}$ when stimulating the skin, it was decided to start with an arbitrarily chosen value of $T_{r}(10 \mathrm{~s})$ and reduce it to the minimum possible. This minimum was chosen on the basis of the criterion defined above. Intuitively, at constant sensitivity, the longer the duration of the $T_{r}$, the greater the recovery of the tactile receptors' sensitivity and the lower the power consumed. However, the higher the value of $T_{r}$, the slower the information transfer. Thus, with the proposed test, the recovery time for the optimum minimum power and maximum sensitivity has been determined experimentally.

Number of pulses per burst. Intuitively, the higher the NPB the higher the sensitivity, and, from Eq. 2, the higher the sensitivity the lower the required power. However, it was also predictable that the higher the $N P B$, the higher the adaptation effect, and therefore the lower the sensitivity and the higher the required power. $N P B$ ranged from 1 to 15 for this testing.

Duty cycle. In the earlier stage of the study a duty cycle $(D C)$ of $1 / 2$ was used. However, from Eq. 2 it can be seen that the power is directly proportional to $D C$ so it would be advantageous to minimize this quantity within the limits imposed by sensitivity thresholds. As an exploratory test, the $D C$ was reduced to $1 / 16$, with a subsequent significant reduction in the power while still allowing the threshold of tactile sensation to be reached. Thus, it was decided to further reduce the $D C$ over the range from $1 / 16$ to $1 / 55(1 / 20,1 / 25,1 / 30,1 / 35,1 / 40,1 / 45,1 / 50,1 / 55)$ in order to detect the $D C$ which still allowed reaching threshold for tactile sensation and minimized the power used. In order to investigate the assumptions stated in the previous paragraphs the following tests were performed:

1. Detection of the first threshold and line sensation threshold for a range of recovery times $(0.1$ to $10 \mathrm{~s})$ at a specific number of pulses/burst $(\mathrm{NBP}=10)$ and two duty cycles $(1 / 2$ and $1 / 16)$.

2. Detection of the first threshold and line sensation threshold for different numbers of pulses/burst (1-15 by steps of 1) at a given recovery time ( $2 \mathrm{~s})$ and duty cycle $(1 / 16)$.

3. Detection of first threshold and line sensation threshold for different duty cycles $(1 / 2-1 / 55)$ at a given range of number of pulses/burst $(9-12)$ and a specific recovery time $(2 \mathrm{~s})$.

Five sighted subjects were used in each test. The subjects wore a straight-line array of five vibrators on their inner forearm. This shape was chosen for the simplicity of 


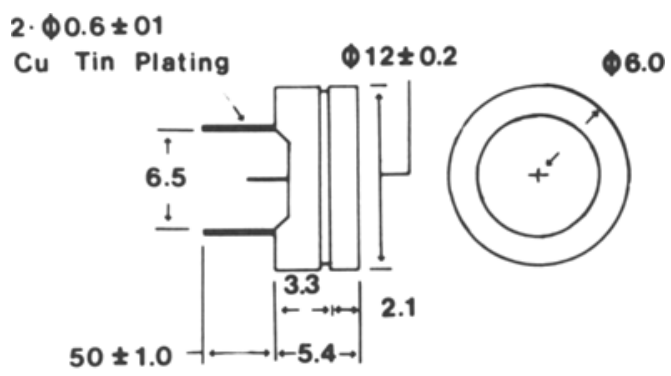

(a)

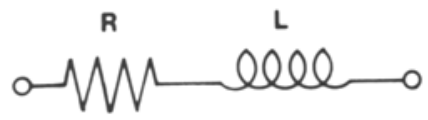

(b)

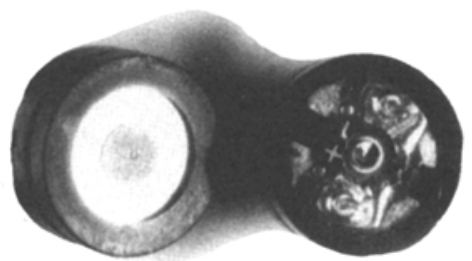

(c)

FIGURE 2. (a) Mechanical diagram, (b) electrical diagram, and (c) actual view of the vibrator used in this study (the mechanical diagram was obtained from the vibrator data sheets provided by Star Micronics, NJ).

the tactile image evoked by the array (i.e., no interpretation was required on the part of the subject). The vibrators were positioned next to each other, thus, the center of the vibrators were spaced $12 \mathrm{~mm}$ apart. The vibrators used are STAR Micronics (Piscataway, NJ) model QMB-105 and their mechanical characteristics are shown in Fig. 2a. The actual vibrator is shown in Fig. 2c.

An electronic circuit was designed to drive the transducers and to allow modification of the values of the stimulus' parameters. In each test the peak current driving the transducers was gradually increased until threshold for tactile sensation was reached. The circuit did not control vibrator depth as the Optacon system which controls the vibrator, to ensure lift-off during part of the cycle, which increases sensitivity. The volunteer subjects were told to report on each type of sensation described above. The test started after a brief familiarization period. No attempt was made to mask the volunteers from hearing the vibrators. It could have been possible that the vibrators were heard before they were felt.

\section{RESULTS}

Test Series No. 1

The first series of tests was performed to determine the value of recovery time $\left(T_{r}\right)$ which minimized the current required to reach the thresholds for tactile sensa- 

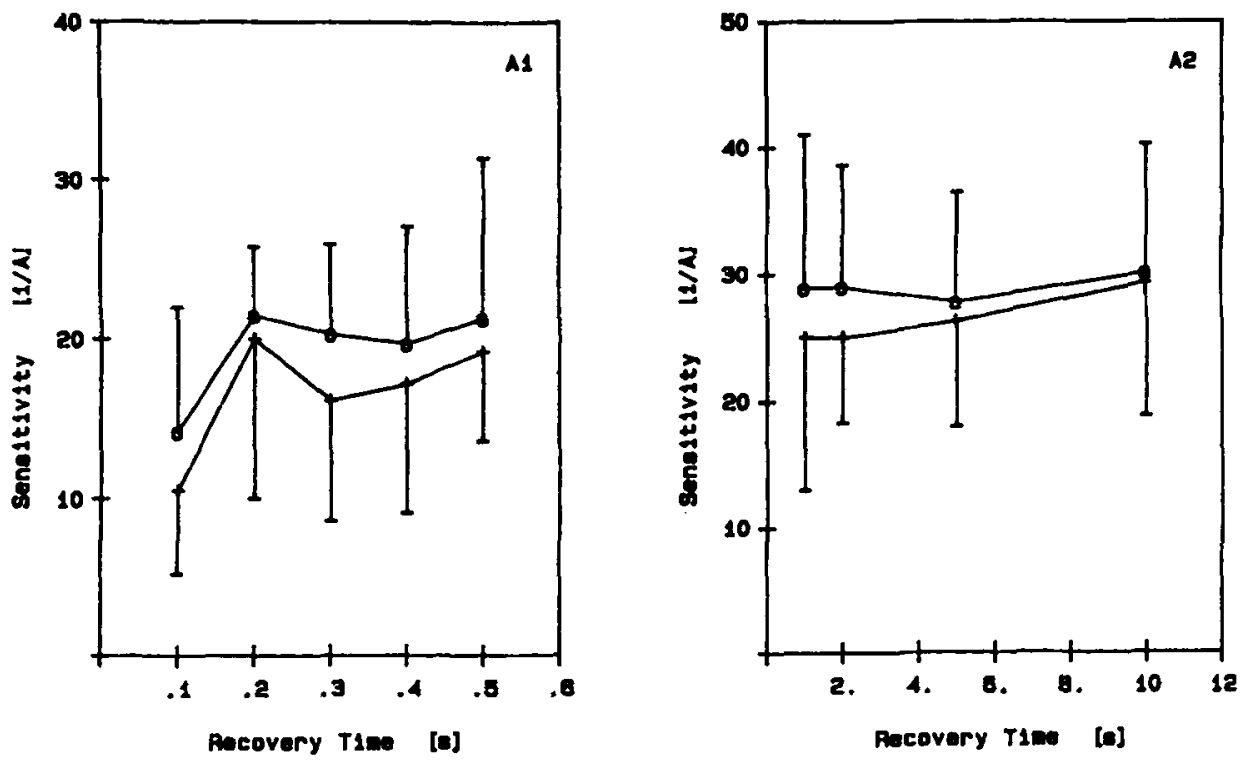

(a)
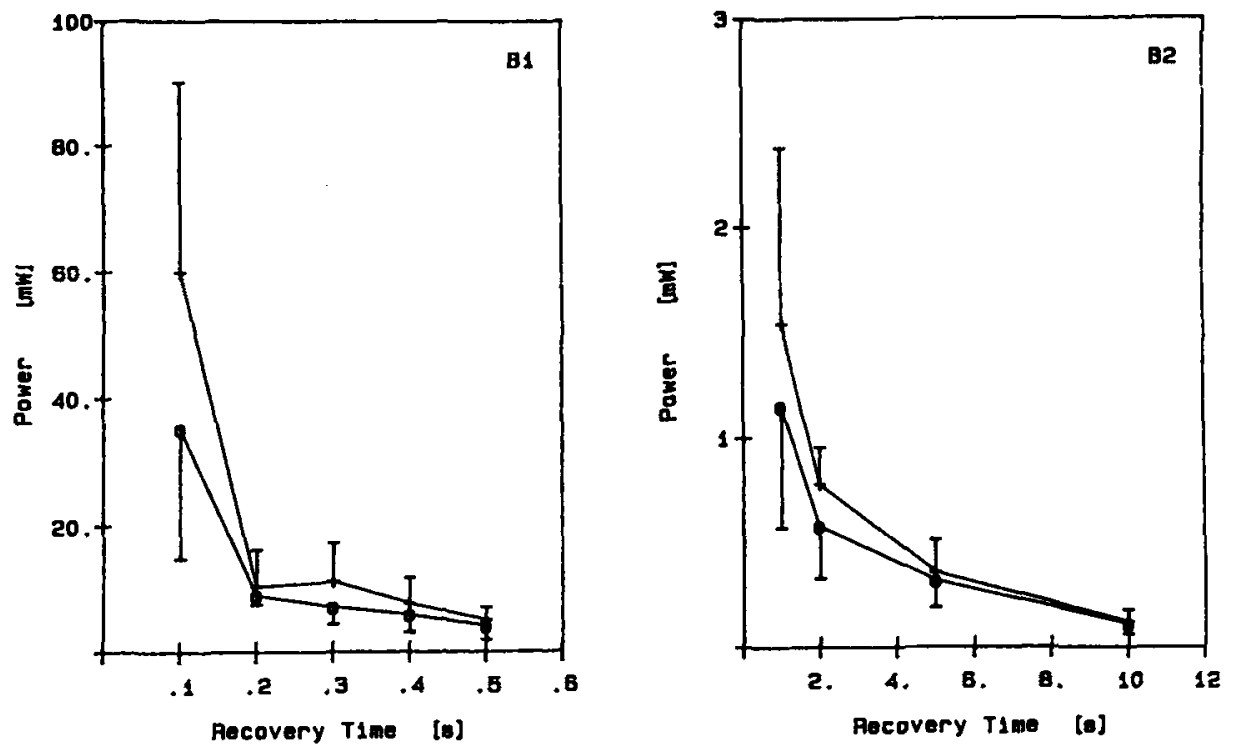

(b)

FIGURE 3. (a) Sensitivity $\left[A^{-1}\right]$ and (b) Power $[\mathrm{mW}]$ (mean $\left.\pm 1 S D\right)$ at the thresholds for first sensation (o) and for line sensation (+) vs. $T$, [s] with $N P B$ equal to 10 and $D C$ equal to $1 / 2 ;(A 1, B 1)$

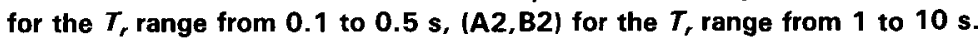

tion. It was assumed that the effects of $T_{r}$ were independent from $N P B, D C$, and $f_{p}$, which is valid since the recovery time is an inherent characteristic of the tactile system, independent from the way the system is stimulated. Two values of $D C$ were used $(1 / 2$ and $1 / 16)$ along with one value of $N P B(10)$. The results for both power and 

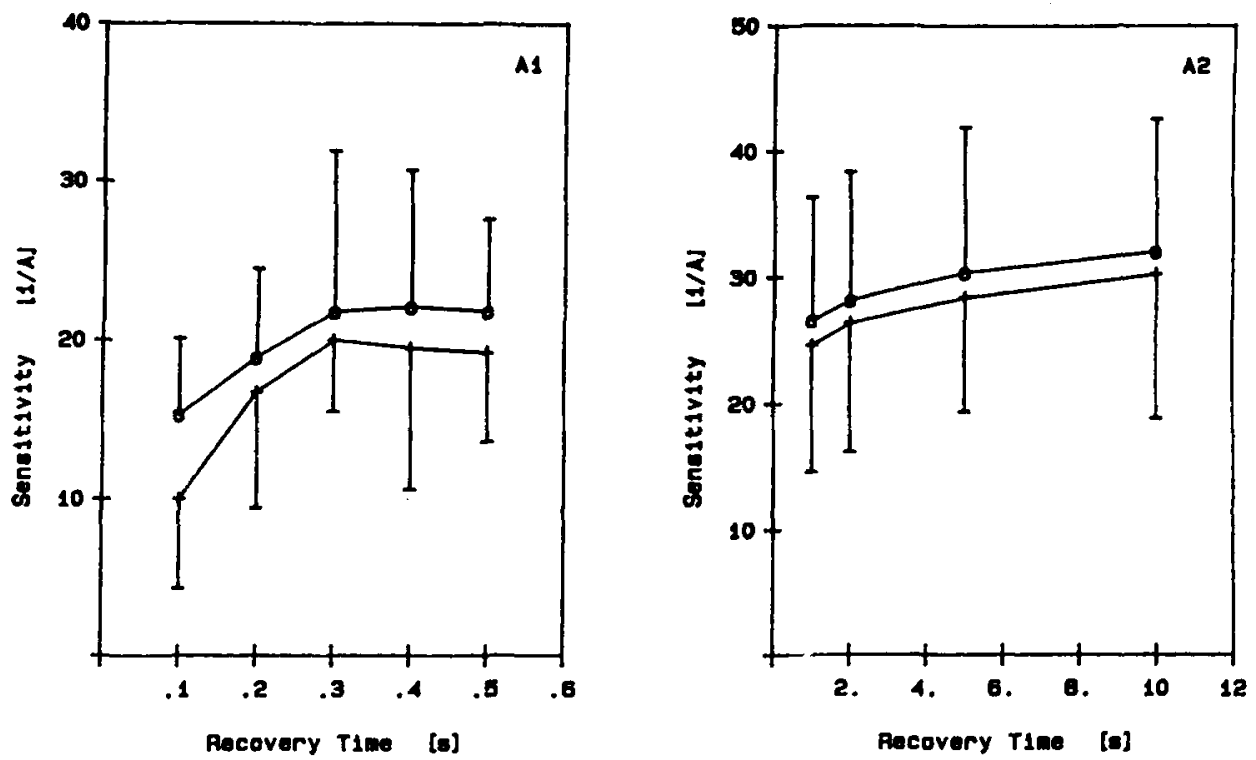

(a)
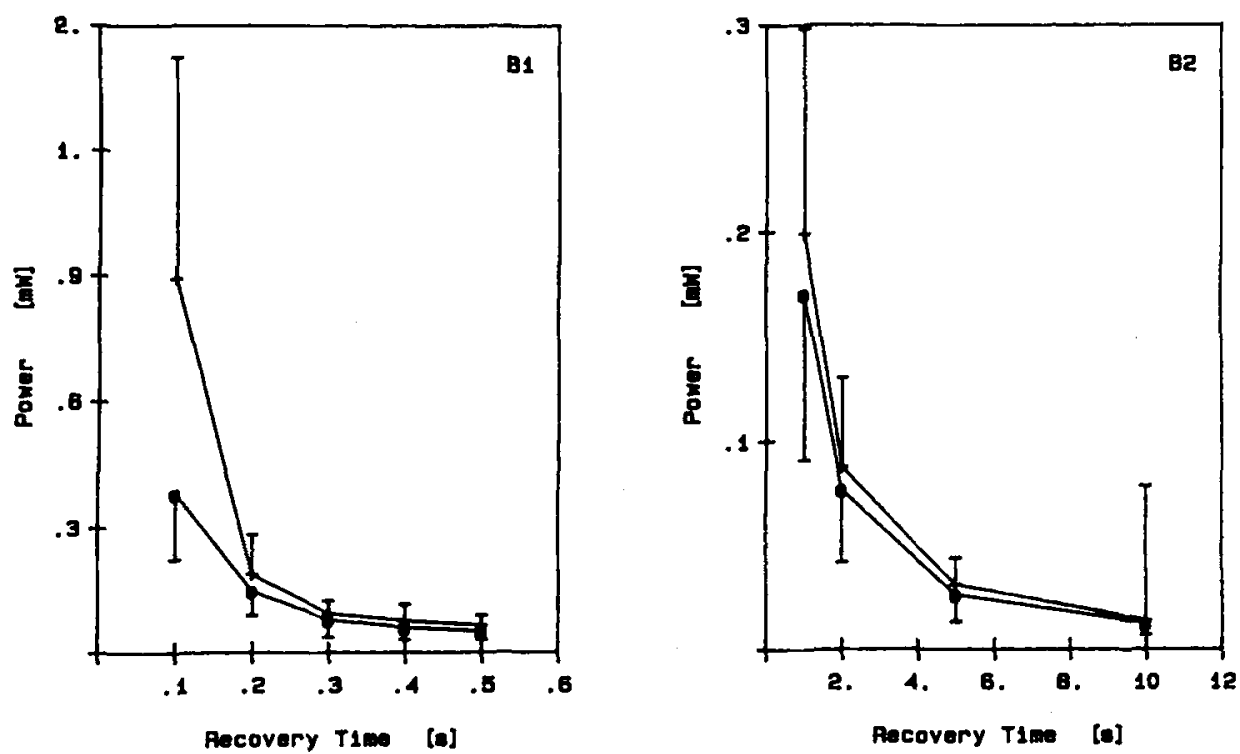

(b)

FIGURE 4. (a) Sensitivity $\left[A^{-1}\right]$ and (b) Power $[\mathrm{mW}]$ (mean $\pm 1 S D$ ) at the thresholds for first sensation $(0)$ and for line sensation $(+)$ vs. $T_{r}$ [s] with $N P B$ equal to 10 and $D C$ equal to $1 / 16 ;(A 1, B 1)$ for the $T$, range from 0.1 to $0.5 \mathrm{~s}$, (A2,B2) for the $T$, range from 1 to $10 \mathrm{~s}$.

sensitivity (mean $\pm 1 S D$ ), for a single vibrator, as functions of recovery time are shown in Figs. 3 and 4.

In Fig. 3 the results are shown for $D C$ equal to $1 / 2$, while in Fig. $4 D C$ is equal to $1 / 16$. The first observation from these results is that with $D C=1 / 16$ the sensi- 
tivity has a net increase of almost $22 \%$ using $T_{r}=1.0$ second with respect to $T_{r}=$ 0.5 second or less. If $T_{r}$ is increased from 2.0 to $10 \mathrm{~s}$, the sensitivity presents another slight increase of $12 \%$ for both $D C$ equal to $1 / 2$ and $D C$ equal to $1 / 16$. As expected, the longer the recovery time, the lower the average power (see Eq. 2), both because the threshold decreases and because the current is on for less total time per stimulus cycle. Moreover, by reducing the $D C$ from $1 / 2$ to $1 / 16$, the power consumption was reduced by an order of magnitude, while the sensitivity was increased by $5 \%$ (at line sensation threshold for recovery time equal to $2 \mathrm{~s}$ ) for $D C$ equal to $1 / 16$.

According to the test criterion a recovery time equal to 5 or $10 \mathrm{~s}$ should have been chosen for the subsequent tests. However, during the test the subjects experienced difficulty in remembering the sensation (if any) produced by the previous stimulation for recovery times greater than $2 \mathrm{~s}$. To avoid this problem, which sometimes led to incorrect detection, and to reduce the slow rate of information transfer produced by a long recovery time, $T_{r}$ was set at $2 \mathrm{~s}$ for the next test series.

\section{Test Series No. 2}

This series of tests was performed to determine a suitable range of the parameter $N P B$ (number of pulses per burst). The $N P B$ was varied through the range from 1 to 15 at $1 N P B$ intervals. The results (mean $\pm 1 S D$ ) are shown in Fig. 5. From this figure it is possible to conclude that beyond $10 N P B$ the sensitivity does not increase with the increase in $N P B$. Actually, the sensitivity has a small decrease in value. On the other hand, the power increases almost proportionally with the increase in $N P B$ which is also a drawback to using a higher $N P B$.

It is possible that the reason behind the sensitivity decrease with increased $N P B$ is adaptation during the burst. Indeed, the subject, before giving his/her response, often needed some time to process the information after the stimulation was applied.

The increase in power with an increase in $N P B$ indicates that the $N P B$ factor in the denominator of Eq. 2 can be considered negligible. Thus, at a given sensitivity, the power is nearly directly proportional to the $N P B$. From Fig. 5a we see that the sensitivity (in the $N P B$ range of 9-12) reaches its maximum and varies no more than $6 \%$ with respect to its maximum value for an $N P B$ equal to 10 (data referred to the line sensation threshold). The power also reaches a relative minimum in this $N P B$ range. Based on these results, it is possible to conclude that values of 9-12 for NPB lead to the highest sensitivity with a relative minimum level of power consumption.

\section{Test Series No. 3}

This test series was performed in order to investigate whether or not it was possible to decrease the duty cycle while still maintaining a high sensitivity. The range of $N P B$ used was from 9 to 12 as suggested by Test Series No. 2. The results were collected for the duty cycle range from $1 / 2$ to $1 / 55$ and plotted with a three-dimensional format in Fig. 6 (mean values). Figure 6 shows the surfaces described by the sensitivity and the power values to the changes of the $N P B$ and the $D C$. The sensitivity is represented by a plane whose function can be considered linear with respect to the $D C$ for all the $N P B(9,10,11,12)$. There are four relative maxima, respectively, at the coordinates: $N P B=9 D C=1 / 2, N P B=10 D C=1 / 2, N P B=10 D C=1 / 25$, and $N P B=12 D C=1 / 2$. The power describes a surface which is clearly not linear 


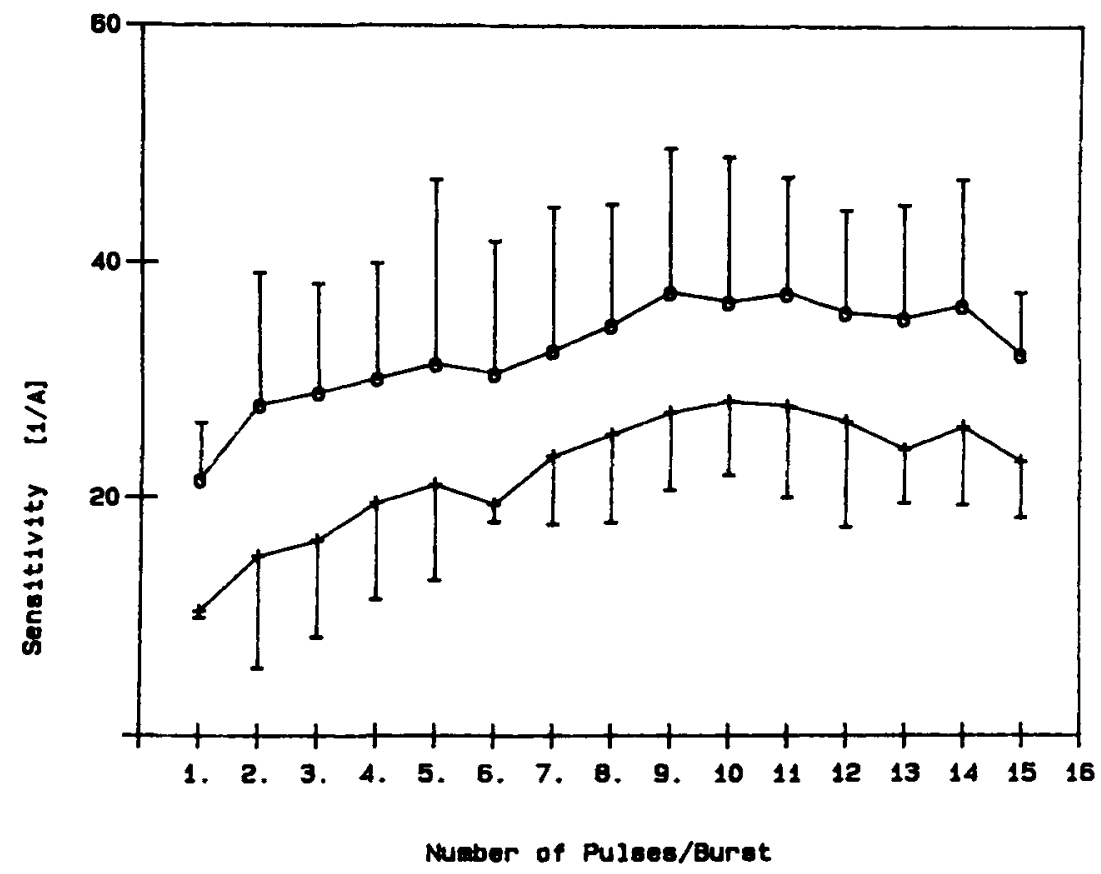

(a).

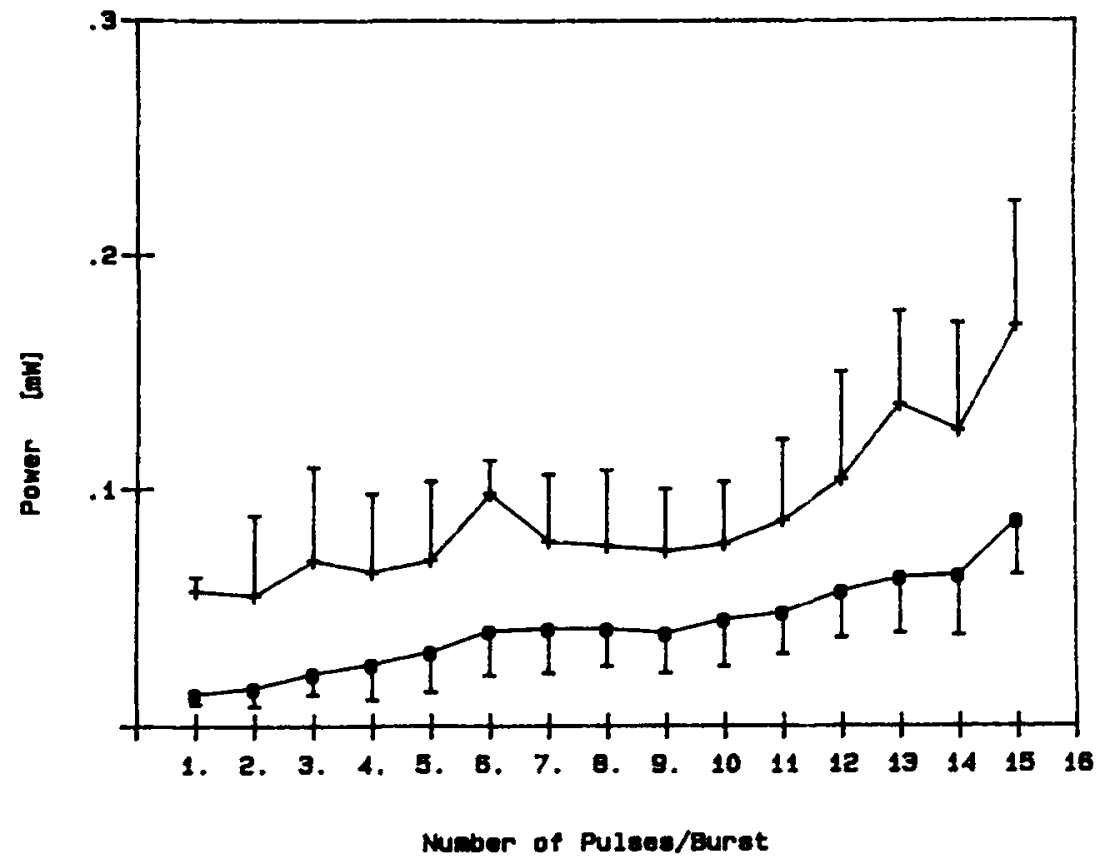

(b)

FIGURE 5. (a) Sensitivity $\left[A^{-1}\right]$ and (b) Power $[\mathrm{mW}]$ (mean $\pm 1 S D$ ) at threshold for first sensation $(0)$ and for line sensation $(+)$ vs. NPB at a $T_{r}$ equal to $2 \mathrm{~s}$ and $D C$ equal to $1 / 16$. 


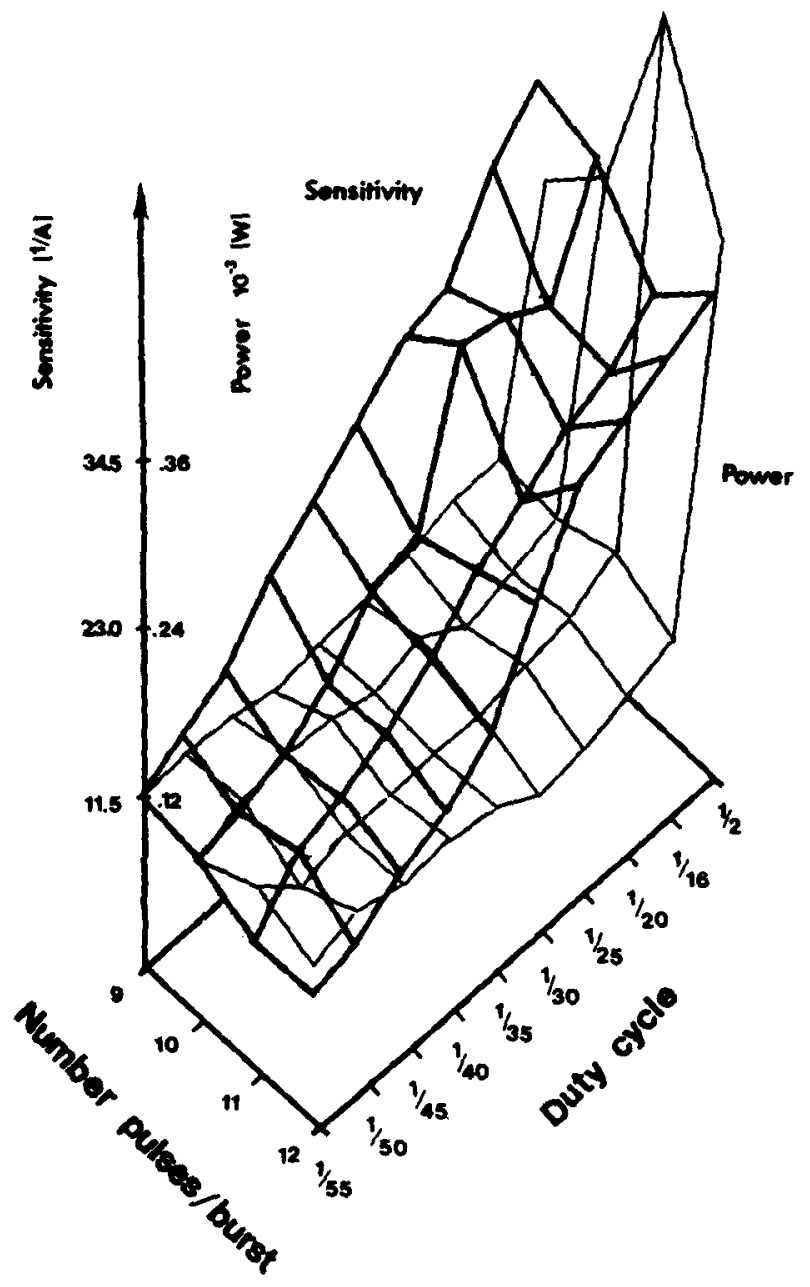

FIGURE 6. Tri-dimensional plot of the (a) sensitivity $\left[A^{-1}\right]$ and (b) Power $[m W]$ (mean values), consumed by a single vibrator, at threshold for line sensation. The sensitivity and the power are plotted vs. $D C$ and $N P B$ per burst.

with respect to the $D C$, defining a parabolic like function whose minimum, for all the $N P B$, is located around $D C=1 / 25$.

From Fig. 6 we can observe that the power is reduced as much as $23 \%$ in changing the $D C$ from $1 / 16$ to $1 / 25$ for $N P B$ equal to 11 . This is accompanied by a sensitivity decrease of less than $9 \%$ compared to its value at a $D C$ equal to $1 / 16$. For values of $D C$ less than $1 / 25$ the power shows a net increase, chiefly because of a decrease in sensitivity. There could be several reasons for this sensitivity decrease: (a) the skin acts, generally, as a low-pass mechanical filter with its own time constant so it does not let the rectangular vibratory pulse pass through completely, or (b) the limiting system could be the vibrator itself, which also has an inherent low-pass transfer function. 
From Fig. 6 it is possible to deduce the combinations of parameters that produce an optimum stimulation pattern. The greater the distance between the sensitivity curve and the power curve, the better the combination of parameters, since the distance indicates the maximum sensitivity at minimum power consumption. This criterion leads us to choose the point corresponding to $N P B$ equal to 10 and $D C$ equal to $1 / 25$. Statistical analysis on the data set of Test Series No. 3 was performed in order to detect statistically the effect of variations in the values of duty cycle and number of pulses per burst. ANOVA analysis (analysis of variance) for repeated measurements shows no significant difference in sensitivity among the four different values $(9,10,11,12)$ of $N P B(p=0.0689)$ while it is strongly significant with respect to the effect of changes in the $D C(p<0.001)$. Similarly, there is no significant difference in power among the $N P B(p>0.05)$ while it is significant with respect to the effect of change in $D C(p<0.001)$.

\section{DISCUSSION}

Results reported in this research (9) summarize the investigation of a 4-dimensional matrix in which the sensitivity was the dependent variable and $T_{r}, N P B$, and $D C$ were the independent variables. A fourth independent variable, frequency of the stimulating pulses, was kept constant throughout the test series. The ranges over which the independent variables were investigated were: 0.1 to $10 \mathrm{~s}$ for the recovery time, 1 to 20 for the number of pulses per burst, and $1 / 2$ to $1 / 55$ for the duty cycle. From the series of tests performed it is possible to conclude that, given the specific conditions under which the tests were run, and for the electromechanical vibrator used, the set of parameters which appear optimum in term of minimum power and maximum sensitivity are: frequency of stimulation, $250 \mathrm{~Hz}$; recovery time, $2 \mathrm{~s}$; number of pulses per burst, 10 ; and duty cycle, $1 / 25$. For these values the sensitivity reaches one of its relative maxima $\left(29.4 \mathrm{~A}^{-1}\right)$ while the power consumed by a single vibrator is at one of its relative minima $(55 \mu \mathrm{W})$. No tests for the effect of variation in duty cycle for values of $N P B$ other than in the range 9-12 were performed. It is our opinion that Test Series No. 2, in which the $N P B$ was varied from 1 to 15 , performed for different duty cycles will lead to the same results. This statement is based on the results of Test Series No. 3. Indeed, in the latter test, the behavior of the sensitivity and power vs. $D C$ was the same for all four values of $N P B$ used.

\section{CONCLUSION}

This experimental study has led to a greater understanding of some of the physiological properties of the tactile system when stimulated with vibrational energy. By analyzing the results of sensitivity and power versus $N P B$ and $D C$ (Test Series No. 3 ), it can be concluded that the tactile system, including the skin layer and the tactile receptors, acts as the combination of a time derivative and integrating system. Indeed, the stimulating pulse can be reduced in width, and still reach the threshold for tactile sensation. This would suggest that the tactile system responds, primarily, to the rate of change of the stimulation pulse and therefore that a very narrow, low energy pulse would be sufficient to elicit the tactile receptors' response (Pacinian corpuscle). On the other hand, when the pulse is too narrow (duty cycle less than $1 / 25$ ) tactile sensation threshold depends on the pulse amplitude due to the non-Pacinian 
corpuscle dependence on the integral of the input pulse, that is, on the total energy in the pulse.

From the results of this study, it appears worthwhile to develop a model of the "vibratile stimulation to mechano-electrical response" system which includes the transfer function of a vibrator, skin layer, PCs, and other tactile receptors. With this combined set of electromechanical and physiological transfer functions it would be possible to compare experimental data with the results predicted by the model. Then, the individual elements of the transfer function could be studied independently to determine which parameters have the greatest impact on the image transfer process in the electromechanical-tactile system. Currently the authors are developing such a model.

\section{REFERENCES}

1. Collins, C.C. Tactile-television-mechanical and electrical image projection. IEEE Trans. Man-Machine Systems. MMS-11:65-71; 1970.

2. Grandori, F.; Pedotti, A. Theoretical analysis of mechano-to-neural transduction in Pacinian corpuscle. IEEE Trans. Biomed. Eng. BME-27:559-565; 1980.

3. Gray, J.A.B.; Sato, M. Properties of the receptor potential in Pacinian corpuscles. J. Physiol. 122: 610-636; 1953.

4. Guyton, A.C. Textbook of medical physiology. Philadelphia, PA: W.B. Saunders; 1976.

5. Loewenstein, W.R.; Skalak, R. Mechanical transmission in a Pacinian corpuscle. An analysis and theory. J. Physiol. 182:346-378; 1966.

6. Moore, J.T. Survey of the mechanical characteristics of skin and tissue in response to vibratory stimulation. IEEE Trans. Man-Machine Systems. MMS-11:79-84; 1970.

7. Nishi, K.; Sato, M. Depolarizing and hyperpolarizing receptor potentials in the non-myelinated nerve terminal in Pacinian corpuscles. J. Physiol. 199:383-396; 1968.

8. Nunziata, E. The optimization of an electromechanical vibrator driving function for tactile stimulation. The Ohio State University; 1985. Thesis.

9. Nunziata, E.; Taige, P.; Lipetz, L.L.; Weed, H.R. A stimulation waveform for a TVSS which minimizes power consumption. Proc. Annu. Conf. IEEE Eng. Med. Biol. Soc.: 856-857; 1987.

10. Rogers, H.C. Choice of stimulator frequency for tactile arrays. IEEE Trans. Man-Machine Systems. MMS-11:5-11; 1970.

11. Sato, M. Response of Pacinian corpuscles to sinusoidal vibration. J. Physiol. 159:391-409; 1961.

12. Verrillo, R.T. Subjective magnitude functions for vibrotaction. IEEE Trans. Man-Machine Systems. MMS-11:19-24; 1970.

13. Verrillo, R.T. Psychophysics of vibrotactile stimulation. J. Acoust. Soc. Am. 77:225-232; 1985.

\section{NOMENCLATURE}

$N P B=$ number of pulses per burst

$T_{r} \quad=$ recovery time

$f_{p} \quad=$ pulse frequency

$T_{s} \quad=$ pulse period, $1 / f_{p}$

$T_{\text {on }}=$ pulse period during which the current flows

$D C=$ pulse duty cycle, $T_{s} / T_{\text {on }}$ 